\title{
Poor predictability of QuantiFERON-TB assay in recipients and donors for tuberculosis development after kidney transplantation in an intermediate-TB- burden country
}

Enkthuya Jambaldorj ${ }^{1+}$, Miyeun Han ${ }^{2+}$, Jong Cheol Jeong ${ }^{3}$, Tai Yeon Koo ${ }^{4}$, Sang $\| \mathrm{Min}^{5}$, Eun Young Song ${ }^{6}$, Jongwon $\mathrm{Ha}^{1,4,5}$, Curie Ahn ${ }^{1,2,4}$ and Jaeseok Yang ${ }^{1,4,5^{*}}$ (D)

\begin{abstract}
Background: Tuberculosis (TB) is a common opportunistic infection after kidney transplantation (KT). The QuantiFERON-TB-Gold In-Tube test (QFT) is widely used for assessing latent TB; however, it is currently unclear whether the pre-KT QFT of the recipient and donor can predict post-KT TB.

Methods: We retrospectively reviewed patients who received KT between January 2009 and December 2015 at Seoul National University Hospital. The QFT was performed in $458 \mathrm{KT}$ recipients and 239 paired living donors, and $138 \mathrm{KT}$ recipients underwent both the QFT and tuberculin skin test (TST). After excluding 12 patients diagnosed as having clinically latent TB, we evaluated whether the QFT of the recipient and donor was predictive for new-onset active TB after $K T$.

Results: The QFT was positive in 101 (22.1\%) recipients and associated with clinically latent TB before KT $(P<0.05)$. However, agreement between the TST and QFT was poor ( $K=0.327$ ). Post-KT TB occurred in 1 of 95 recipients with a positive QFT, and 2 cases of TB occurred among 351 patients with a negative or indeterminate QFT. The incidence of TB was 242 cases/100,000 person-years among $446 \mathrm{KT}$ recipients with a median follow-up of 30. 2 months. The QFT of recipients could not predict post-KT TB in Poisson regression analysis (relative risk [RR], 1.847; 95\% confidence interval $[\mathrm{Cl}], 0.168-20.373 ; P=0.616)$. Of 234 living donor-recipient pairs, the QFT of the recipient (RR, 5.012; 95\% Cl, 0.301-83.430; $P=0.261$ ) and QFT of the donor (RR, $1.758 ; 95 \% \mathrm{Cl}, 0.106-29.274 ; P=0.694)$ could not predict post-KT TB.
\end{abstract}

Conclusion: The QFT of recipients or living donors pre-KT cannot predict the short-term development of post-KT TB in an intermediate TB-burden country.

Keywords: Donor, Kidney transplantation, Recipient, Tuberculosis

\footnotetext{
*Correspondence: jcyj@@dreamwiz.com

†Equal contributors

${ }^{1}$ Transplantation Research Institute, Seoul National University Hospital, 101

Daehak-ro, Jongno-gu, Seoul 110-744, Republic of Korea

${ }^{4}$ Transplantation Center, Seoul National University Hospital, Seoul, Korea

Full list of author information is available at the end of the article
} 


\section{Background}

Tuberculosis (TB) is a common opportunistic infection after transplantation. The incidence of TB among transplant recipients is 20-74 times higher than that in the general population [1], and TB after transplantation can adversely affect graft viability and patient survival [2]. The most common cause of TB in kidney transplantation (KT) recipients is reactivation of latent TB. Therefore, screening and treating active or latent TB in all transplant candidates is widely recommended [2-4].

History taking, physical examination, and chest radiography should be performed to assess for TB before transplantation. To make a diagnosis of latent $\mathrm{TB}$, the tuberculin skin test (TST) or interferon (IFN)- $\gamma$ release assay (IGRA) is used. However, the Korean guidelines for $\mathrm{TB}$, which were revised in 2014, recommend the use of IGRA alone or TST combined with IGRA for diagnosing latent TB in patients with end-stage renal disease (ESRD), because TST may yield a false positive result in those with a previous Bacillus Calmette-Guérin (BCG) vaccination or a false negative in patients with $\operatorname{ESRD}[5,6]$.

The TB burden of South Korea is decreasing, but the country is still classified as having an intermediate TB burden, with an annual TB incidence of 86/100,000 person-years in 2015 [7] and a TB incidence after KT of 0.283 cases/100 patient-years in a previous study [8]. The QuantiFERON-TB-Gold In-Tube test (QFT) (Cellestis Ltd., Carnegie, Victoria, Australia) was introduced at our center in 2009. However, because the significance of QFT results in immunosuppressed patients was not sufficiently validated, we did not administer anti-TB medications to patients based on the results of QFT alone, unless there was evidence of clinically latent TB, which includes abnormal chest radiograph findings or a history of $\mathrm{TB}$ without complete anti-TB medication and recent close contact with individuals with active TB. The predictive ability of the QFT for post-KT TB in KT recipients is still unclear; therefore, we investigated the efficacy of using the QFT pre-KT in recipients and donors to predict the subsequent development of TB after KT.

\section{Methods}

\section{Study population}

This study was a retrospective, single-center study. Nine hundred seventy-seven cases of KT were performed from January 1, 2009 to December 31, 2015 at Seoul National University Hospital. KT recipients who underwent the QFT were enrolled in this study. The association between clinical latent TB and QFT or TST was analyzed with the recipient who underwent the QFT and TST. The predictability of the QFT of recipients and donors for post-KT TB was analyzed after excluding recipients with isoniazid prophylaxis. This study was approved by our institutional review board (H-1310-
069-527), and the need for informed consent was waived because of its retrospective design.

\section{Data collection}

The demographic characteristics and laboratory results of $\mathrm{KT}$ recipients and donors were retrospectively obtained from medical records. Age, gender, body weight, KT time, donor type, donor age, donor gender, comorbid disease including a history of TB infection and previous BCG vaccination, post-transplant medications including immunosuppressants, and laboratory data including QFT and chest radiographs were collected. TB development after $\mathrm{KT}$ was determined by microbiological confirmation and the initiation of anti-TB medication. Clinical latent TB was defined as (1) an inadequate history of anti-TB medication with a history of TB infection or an inactive TB lesion on a chest radiograph, (2) close contact with a person with pulmonary TB within the past year, and (3) recent conversion of the TST to a positive status $[9,10]$.

\section{Laboratory testing (IGRAs and TST)}

Peripheral blood samples for the QFT were collected, transferred to the laboratory, and processed within $3 \mathrm{~h}$ according to the manufacturer's instructions [11]. The QFT results were classified as positive, negative, or indeterminate, as previously described : positive if the response to the specific antigens was $\geq 0.35 \mathrm{IU} / \mathrm{mL}$, regardless of the value of the positive control; negative if the response to the specific antigens was $<0.35 \mathrm{IU} / \mathrm{mL}$ and the IFN- $\gamma$ level of the positive control was $\geq 0.5 \mathrm{IU} / \mathrm{mL}$; and indeterminate if both antigen-stimulated samples were $<0.35 \mathrm{IU} / \mathrm{mL}$ and the value of the positive control was $<0.5 \mathrm{IU} / \mathrm{mL}[12]$.

TST was performed by trained nurses within 1 week after the QFT. Two tuberculin units of purified protein derivative RT23 (Statens Serum Institute, Copenhagen, Denmark) were injected intradermally on the volar side of the forearm contralateral to the patient's vascular access (the Mantoux technique). The diameter of induration was measured $48 \mathrm{~h}$ after administration. The test was considered positive if the diameter of induration was $\geq 10 \mathrm{~mm}$.

\section{Statistical analysis}

Categorical variables are presented as frequencies, and continuous values are expressed as the mean \pm standard deviation. Proportions were compared using the chisquare test, and continuous variables were compared using the Student t-test. Poisson regression models were used to estimate the relative risk (RR) and 95\% confidence interval (CI). A P-value $<0.05$ was considered statistically significant. All statistical analyses were conducted using SPSS, version 22.0 (SPSS Inc., Chicago, IL). 


\section{Results}

\section{Characteristics of the study population}

The QFT was performed in $458 \mathrm{KT}$ recipients (living donor KT, 303; deceased donor KT, 155). Baseline characteristics for the 458 recipients are shown in Table 1. Patients' mean age was $44.5 \pm 15.4$ years, and 300 (65.4\%) were men. Twenty-one (4.6\%) KT recipients had a TB history, and all of them received adequate anti-TB medication. Seventeen (3.7\%) patients showed radiologic evidence of previous TB, such as a calcified granuloma or nodule (six patients), nodular opacity (8), and other (3). However, 12 had not received adequate anti-TB medication. Hypertension and diabetes were found in $377(82.3 \%)$ and $110(24.0 \%)$ patients, respectively.

Of 458 recipients with QFT results, 239 matched living donors underwent the QFT. Clinical information for the 239 paired recipients and living donors are shown in Table 1. The mean age of the 239 recipients was $40.6 \pm$ 16.1 years, and 157 (65.7\%) were men. Seven (2.9\%) had a history of TB, and seven (2.9\%) had inactive TB lesions on a chest radiograph. The mean age of the 239 paired donors was $45.9 \pm 10.2$ years, and $103(43.1 \%)$ were men. Nineteen (7.9\%) donors had a history of TB, and 14 (5.9\%) had inactive TB lesions on a chest radiograph. Nineteen (7.9\%) donors had hypertension and $1(0.4 \%)$ had diabetes mellitus.

\section{Association of the diagnostic tests with clinically latent TB infection}

Of 458 recipients with QFT results, 138 also underwent TST. The association of the diagnostic tests with clinically latent TB was analyzed in 138 recipients who underwent both tests (Table 2). Among $458 \mathrm{KT}$ recipients with QFT results, 101 (22.1\%) showed positive QFT results, and a positive QFT result was associated with radiologic evidence of previous TB $(P<0.001)$, a history of previous TB $(P<0.05)$, and overall clinically latent TB $(P<0.05)$.

Among $138 \mathrm{KT}$ recipients who underwent QFT and TST, the QFT tended to be associated with a chest radiographic lesion, history of previous $\mathrm{TB}$, and clinically latent $\mathrm{TB}$ despite the absence of statistical significance (Table 2). However, TST was not associated with clinically latent TB, and agreement between TST and QFT in these $138 \mathrm{KT}$ recipients was poor $(\mathrm{K}=0.327)$.

\section{Observations on the development of TB after KT}

After excluding 12 patients with clinically latent TB who received isoniazid treatment, 446 cases were analyzed to determine whether QFT was predictive for post-KT TB development. The predictive ability of QFT for post-KT TB development was also analyzed in 234 living donorrecipient pairs after excluding 5 recipients with clinically latent $\mathrm{TB}$ who received isoniazid treatment (Fig. 1a and b).

Active TB occurred in 3 of $446 \mathrm{KT}$ recipients, 1 with a positive QFT result and 2 with negative or indeterminate results, at a median follow-up of 30.2 months (Fig. 1a). The incidence of TB was estimated to be $0.242 / 100$ person-years; $0.360 / 100$ person-years in the positive QFT group and $0.208 / 100$ person-years in the negative or indeterminate QFT group (Table 3).

Table 1 Baseline clinical characteristics of study population

\begin{tabular}{|c|c|c|c|}
\hline & \multirow[t]{2}{*}{ Recipients ( $n=458)$} & \multicolumn{2}{|c|}{ Recipient-donor pairs ( $n=239$ ) } \\
\hline & & Recipients $(n=239)$ & Donors $(n=239)$ \\
\hline Age, year $( \pm S D)$ & $44.5 \pm 15.4$ & $40.6 \pm 16.1$ & $45.9 \pm 10.2$ \\
\hline Male gender & $300(65.5)$ & $157(65.7)$ & $103(43.1)$ \\
\hline History of TB infection & $21(4.6)$ & $7(2.9)$ & $19(7.9)$ \\
\hline History of close contact with active TB & $0(0.0)$ & $0(0.0)$ & $0(0.0)$ \\
\hline Abnormal chest radiography & $17(3.7)$ & $7(2.9)$ & $14(5.9)$ \\
\hline History of adequately treated TB & $5(1.1)$ & $2(0.8)$ & $1(0.4)$ \\
\hline History of untreated TB & $12(2.6)$ & $5(2.1)$ & $13(5.4)$ \\
\hline \multicolumn{4}{|l|}{ Underlying disease } \\
\hline Hypertension & $377(82.3)$ & $184(77.0)$ & $19(7.9)$ \\
\hline Diabetes mellitus & $110(24.0)$ & $49(20.5)$ & $1(0.4)$ \\
\hline History of malignancy & $32(7.0)$ & $9(3.8)$ & $0(0.0)$ \\
\hline COPD & $3(0.7)$ & $1(0.4)$ & $0(0.0)$ \\
\hline \multicolumn{4}{|l|}{ Laboratory findings } \\
\hline WBC count (cells/ $\mu \mathrm{L})$ & $6850.6 \pm 3617.4$ & $7004.2 \pm 3633.4$ & $6175.2 \pm 1765.9$ \\
\hline Albumin $(\mathrm{g} / \mathrm{dL})$ & $4.0 \pm 1.6$ & $3.9 \pm 0.6$ & $4.4 \pm 0.4$ \\
\hline Cholesterol (mg/dL) & $160.4 \pm 75.9$ & $159.6 \pm 97.9$ & $197.9 \pm 61.6$ \\
\hline
\end{tabular}


Table 2 The association between Tuberculin skin test or QuantiFERON results and latent tuberculosis

\begin{tabular}{|c|c|c|c|c|c|c|}
\hline & \multicolumn{2}{|c|}{ QuantiFERON $(n=458)$} & \multicolumn{2}{|c|}{ QuantiFERON $(n=138)$} & \multicolumn{2}{|c|}{$\mathrm{TST} \geq 10 \mathrm{~mm}(n=138)$} \\
\hline & Positive & $\overline{\text { Negative/indeterminate }}$ & Positive & $\overline{\text { Negative/indeterminate }}$ & Positive & Negative \\
\hline Number (percent, \%) & $101(22.1)$ & $357(77.9)$ & $36(26.1)$ & $102(73.9)$ & $18(13.0)$ & $120(87.0)$ \\
\hline Abnormal chest radiography & $10(9.9)$ & $7(2.0)^{* *}$ & $4(11.1)$ & $3(2.9)$ & $0(0.0)$ & $7(5.8)$ \\
\hline History of previous TB & $10(9.9)$ & $11(3.1)^{*}$ & $3(8.3)$ & $3(2.9)$ & $2(11.1)$ & $4(2.9)$ \\
\hline Clinical latent TB & $6(5.9)$ & $6(1.7)^{*}$ & $3(8.3)$ & $3(2.9)$ & $0(0.0)$ & $6(5.0)$ \\
\hline
\end{tabular}

TB tuberculosis, TST tuberculin skin test

${ }^{*} P<0.05,{ }^{* *} P<0.001$ by Chi-square test

When 234 KT recipients with donor QFT were evaluated, 2 active TB cases were noted, one from a recipient QFT (+)/donor QFT (-) pair and another from a recipient QFT (-)/donor QFT (+) pair (Fig. 1b). The incidences of $\mathrm{TB}$ in each situation are shown in Table 3.

The clinical characteristics of 3 patients with post-KT TB are summarized in Table 4. All cases were livingdonor $\mathrm{KT}$ without desensitization, and post-KT times to TB diagnosis were 38,10 , and 7 months after KT. One patient had pulmonary TB, and 2 patients had extrapulmonary TB.

\section{The predictive ability of QuantiFERON for post-transplant}

TB

We performed Poisson regression analysis to assess the predictive ability of the QFT for post-KT TB development (Table 5). The analysis of 446 recipients with QFT showed that QFT positivity did not predict post-KT TB
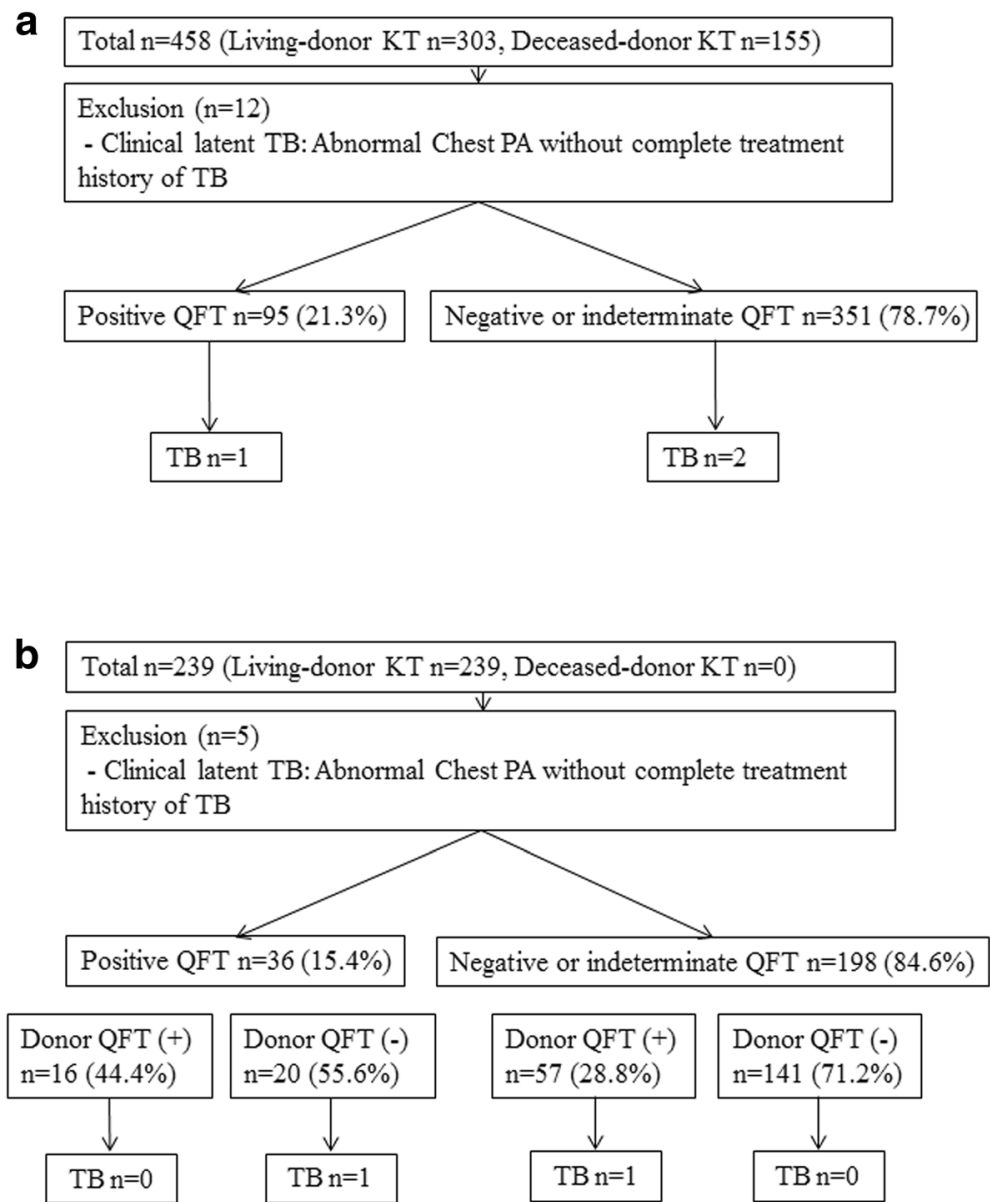

Fig. 1 The development of TB after KT. a Four hundred fifty-eight KT recipients who underwent a QFT. $\mathbf{b}$ Two hundred thirty-nine KT recipients with a paired living donor with a QFT. KT, kidney transplantation; TB, tuberculosis; QFT, QuantiFERON-TB-Gold In-Tube test 
Table 3 Tuberculosis incidence according to donor/recipient QuantiFERON status

\begin{tabular}{|c|c|c|c|c|c|}
\hline \multirow[t]{2}{*}{ Variables } & \multicolumn{5}{|c|}{ TB incidence rates } \\
\hline & No. of patients & No. of TB cases & No. of person-years & TB rate per 100,000 person-years & $95 \% \mathrm{Cl}$ \\
\hline Total patients & 446 & 3 & 1238.7 & 242.2 & $49.9,708.8$ \\
\hline Positive QFT & 95 & 1 & 277.6 & 360.2 & $9.1,2007.1$ \\
\hline Negative or indeterminate QFT & 351 & 2 & 961.1 & 208.1 & $25.2,751.7$ \\
\hline Living donor-recipient pairs & 234 & 2 & 551.8 & 362.5 & $43.9,1309.3$ \\
\hline Recipient QFT (+)/Donor QFT (+) & 16 & 0 & 51.6 & 0 & $0,7149.9$ \\
\hline Recipient QFT (+)/Donor QFT (-) & 20 & 1 & 48.2 & 2074.7 & $52.5,11559.4$ \\
\hline Recipient QFT (-)/Donor QFT (+) & 57 & 1 & 134.9 & 741.3 & $18.8,4130.2$ \\
\hline Recipient QFT (-)/Donor QFT (-) & 141 & 0 & 317.1 & 0 & $0,1163.3$ \\
\hline
\end{tabular}

Cl confidence interval, No number, QFT QuantiFERON test, TB tuberculosis

development (RR, 1.847; 95\% CI, 0.168-20.373; $P=0.616$ ). When the 234 donor-recipient pairs were analyzed, neither recipient QFT positivity (RR, 5.012; 95\% CI, 0.30183.430; $P=0.261$ ) nor donor QFT positivity (RR, 1.758; 95\% CI, 0.106-29.274; $P=0.694$ ) could predict post-KT TB development (Table 5).

\section{Discussion}

In this study, the QFT was positive in $22 \%$ of KT recipients, and recipient QFT positivity was associated with clinically latent $\mathrm{TB}$ before $\mathrm{KT}$. We showed that that the incidence of post-KT TB was 0.242 cases $/ 100$ personyears among $446 \mathrm{KT}$ recipients at a median follow-up of 30.2 months and that neither recipient QFT positivity nor donor QFT positivity could predict post-KT TB development.

Consistent with previous reports [12, 13], the QFT was significantly associated with latent $\mathrm{TB}$, whereas the TST showed no such association in patients with ESRD. Moreover, agreement between the QFT and TST was poor. Overall, our study demonstrated that the QFT is a better diagnostic test for latent TB than TST in KT candidates. Treatment of latent $\mathrm{TB}$ is recommended for solid organ transplant recipients with a positive TST or IGRA result [2-4]. However, the actual benefit of this practice is still unknown [2, 14]. IGRA yields a high rate of false positives [15], and some TB breakthrough cases developed despite isoniazid prophylaxis [16, 17]. Moreover, concerns about drug toxicity and resistance may not justify routine isoniazid prophylaxis for recipients with a positive IGRA result in a country with high-tointermediate TB burden, unless the predictive capacity of IGRA for post-TB development is confirmed.

Several studies have been performed to validate the diagnostic usefulness of the IGRA test for active TB. Pooled sensitivity for active TB was $80 \%$ for the QFT and $81 \%$ for the TB-specific ELISPOT assay (T-SPOT), and pooled specificity of the QFT for active TB was 79 and 59\% for T-SPOT [18]. However, the diagnostic performance for extrapulmonary TB that is more common in KT patients $[19,20]$ was slightly lower; pooled sensitivity was $72 \%$ for the QFT and $90 \%$ for T-SPOT, and

Table 4 Clinical characteristics of post-transplant tuberculosis cases

\begin{tabular}{llll}
\hline & Case 1 & Case 2 & Case 3 \\
\hline Age range & $40-50$ & $40-50$ & $40-50$ \\
Previous treatment (duration) & Preemptive & HD (4 months) & HD (1 month) \\
LDKT or DDKT & LDKT & LDKT & LDKT \\
Recipient QFT & Negative & Positive & Negative \\
Donor QFT & Positive & Negative & N/A \\
Recipient TST & N/A & N/A & 0 mm \\
Desensitization & No & No & No \\
Induction immunosuppression & Basiliximab & Basiliximab & Basiliximab \\
Maintenance immunosuppression & Steroid, tacrolimus, MMF & Steroid, tacrolimus, MMF & Steroid, tacrolimus, MMF \\
Time to TB diagnosis after KT & 38 months & 10 months & 7 months \\
Type of TB & TB lymphadenopathy & TB colitis & Pulmonary TB \\
\hline
\end{tabular}

$F$ female, $H D$ hemodialysis, $K T$ kidney transplantation, $L D K T$ living-donor kidney transplantation, $M$ male, $M M F$ mycophenolate mofetil, $N / A$, not applicable, TB tuberculosis 
Table 5 Poisson regression analysis for predictablity of QuantiFERON test for post-transplant tuberculosis

\begin{tabular}{llll}
\hline & RR & $95 \%$ Cl & $P$ value \\
\hline Model 1 & & & \\
$\quad$ Recipient QFT (+) & 1.847 & $0.168,20.373$ & 0.616 \\
Model 2 & & & \\
$\quad$ Recipient QFT (+) & 5.012 & $0.301,83.430$ & 0.261 \\
$\quad$ Donor QFT (+) & 1.758 & $0.106,29.274$ & 0.694 \\
\hline
\end{tabular}

$\mathrm{Cl}$ confidence interval, QFT QuantiFERON test, $R R$ relative risk A total of 446 recipients without clinical latent TB were included in model 1 and 234 donor-recipient pairs with QFT results were included in model 2

pooled specificity was $82 \%$ for the QFT and $68 \%$ for TSPOT [21]. IGRA also showed a good negative predictive value (NPV) but a very poor positive predictive value (PPV) for progression to active TB [2]. Pre-transplant TSPOT failed to show significant predictive potential for post-transplant TB development [9]. In parallel, the PPV and NPV of the QFT to predict TB in our results were 1.05 and $99.43 \%$, respectively. Although the QFT seems to be slightly more specific than T-SPOT, our results showed that the QFT cannot sufficiently predict posttransplant TB development. Taken together, we propose using isoniazid prophylaxis for all patients with clinically latent TB. Regarding the cases of positive IGRA without evidence of clinically latent $\mathrm{TB}$, routine isoniazid prophylaxis may not be needed for $\mathrm{KT}$ patients in intermediate to high TB-burden countries until we can obtain contradictory evidence from large-scale, long-term follow-up studies, due to a low PPV of IGRA for active TB development and concerns about drug toxicity and resistance.

Interestingly, two cases of $\mathrm{TB}$ developed in recipients with negative QFT results. This suggested that the QFT, just like T-SPOT, is not adequately sensitive in immunosuppressed hosts [9]. De novo post-KT TB infection can also occur in countries with a high-to-intermediate TB burden. Yet, latent TB in the donor may be transmitted to the recipient after KT [22]. Actually, one case of TB in a patient with a negative QFT result received kidneys from a QFT-positive donor. Organ donation from donors with active TB is generally contraindicated; however, there is some controversy concerning isoniazid treatment for donors or recipients when the donor has positive IGRA results. Some guidelines recommend chemoprophylaxis for donors with a positive TST/IGRA before donation [22]; however, it is challenging to treat all donors with positive IGRA results for the same reason as recipients. In our study, $31 \%$ of donors were QFT positive, and the QFT of the donor could not predict the development of TB. Although treatment of latent TB for KT recipients with QFT-positive donors is recommended in low TB-burden countries, routine treatment is still controversial in high-to-intermediate TB-burden countries, because drug toxicity and resistance may outweigh the benefits of treatment [22]. Further large-scale, long-term follow-up studies are needed to confirm the necessity of treatment based on donor QFT. Before confirmative evidence is available, a careful approach is needed according to a center-specific policy, and we recommend close monitoring of KT patients with a positive donor IGRA result without routine treatment in our situation.

There were some limitations to our study. First, our study was retrospective; therefore, the exact history of contact with active TB patients was difficult to ascertain. Second, the QFT or TST was not performed for all recipients. The QFT was preferentially performed in adult or living donor KT patients. However, when we analyzed the incidence rate of post-KT $\mathrm{TB}$ in patients at our center who did not undergo the QFT and in the study population of the KNOW-KT [23], there was no significant difference in the incidence rate between our study population and either group. Next, the follow-up duration was relatively short and the study population was small for the adequate assessment of the predictive usefulness of post-transplant TB development. Nevertheless, the present study is the first study to assess the predictive potential of pre-KT QFT in recipients and donors for post-KT TB development in an intermediate TB-burden country. Larger scale studies with a longterm follow-up are needed to verify our findings.

\section{Conclusions}

The QFT is more useful than the TST for diagnosing a latent TB infection in KT candidates. However, the QFT in both recipients and paired living donors may not predict the short-term development of post-transplant TB in an intermediate-TB-burden country.

\section{Abbreviations}

BCG: Bacillus Calmette-Guérin; Cl: Confidence interval; ESRD: End-stage renal disease; IFN: Interferon; IGRA: Interferon- $\gamma$ release assay; KT: Kidney transplantation; NPV: Negative predictive value; PPV: Positive predictive value; QFT: QuantiFERON TB Gold In-Tube test; RR: Relative risk; TB: Tuberculosis; T-SPOT: Tuberculosis-specific ELISPOT assay; TST: Tuberculin skin test

\section{Acknowledgements}

Not applicable.

\section{Funding}

This study was supported by a research fund (2015) from the Korean Society for Transplantation.

\section{Availability of data and materials}

The datasets used during the current study are available upon request from the corresponding author.

\section{Authors' contribution}

EJ, JCJ, and JY participated in the research design. EJ, MH, JCJ, TYK, SIM, EYS, $J H, C A$, and $J Y$ participated in data collection and analysis. $\mathrm{MH}$, and $\mathrm{JY}$ participated in writing of the paper. All authors read and approved the final manuscript. 


\section{Competing interests}

The authors declare that they have no competing interests.

\section{Consent for publication}

Not applicable.

\section{Ethics approval and consent to participate}

This study was approved by the institutional review board of Seoul National University Hospital (H-1310-069-527). The need for informed consent was waived because of its retrospective design

\section{Publisher's Note}

Springer Nature remains neutral with regard to jurisdictional claims in published maps and institutional affiliations.

\section{Author details}

${ }^{1}$ Transplantation Research Institute, Seoul National University Hospital, 101 Daehak-ro, Jongno-gu, Seoul 110-744, Republic of Korea. ${ }^{2}$ Department of Internal Medicine, Seoul National University College of Medicine, Seoul, Korea. ${ }^{3}$ Department of Internal Medicine, Ajou University School of Medicine, Suwon, Korea. ${ }^{4}$ Transplantation Center, Seoul National University Hospital, Seoul, Korea. ${ }^{5}$ Department of Surgery, Seoul National University College of Medicine, Seoul, Korea. ${ }^{6}$ Department of Laboratory Medicine, Seoul National University College of Medicine, Seoul, Korea.

Received: 29 December 2016 Accepted: 9 March 2017 Published online: 14 March 2017

\section{References}

1. Munoz P, Rodriguez C, Bouza E. Mycobacterium tuberculosis infection in recipients of solid organ transplants. Clin Infect Dis. 2005:40(4):581-7.

2. Munoz L, Santin M. Prevention and management of tuberculosis in transplant recipients: from guidelines to clinical practice. Transplantation. 2016;100(9):1840-52

3. Kidney Disease-Improving Global Outcomes (KDIGO) Transplant Work Group. KDIGO clinical practice guideline for the care of kidney transplant recipients. Am J Transplant. 2009:9(Suppl 3):S1-155.

4. Bumbacea D, Arend SM, Eyuboglu F, Fishman JA, Goletti D, Ison MG, et al. The risk of tuberculosis in transplant candidates and recipients: a TBNET consensus statement. Eur Respir J. 2012;40(4):990-1013.

5. Joint Committee for the Revision of Korean Guidelines for Tuberculosis, Korean Centers for Disease Control and Prevention. Korean guidelines for tuberculosis. 2nd ed. Seoul and Cheongwon: Joint Committee for the Revision of Korean Guidelines for Tuberculosis, Korea Centers for Disease Control and Prevention; 2014

6. Lee SH. Diagnosis and treatment of latent tuberculosis infection. Tuberc Respir Dis (Seoul). 2015;78(2):56-63.

7. World Health Organization. Global tuberculosis report 2015. Geneva: World Health Organization; 2015.

8. Jeong JC, Koo TY, Jeon HJ, Park HC, Ryu HJ, Lee JP, et al. Utility of QuantiFERON-TB assay for prediction of tuberculosis development in kidney transplant patients in an intermediate-tuberculosis-burden country: lack of evidence for enhanced prediction for short-term tuberculosis development. Transplant Proc. 2014;46(2):583-7.

9. Kim SH, Lee SO, Park IA, Kim SM, Park SJ, Yun SC, et al. Isoniazid treatment to prevent TB in kidney and pancreas transplant recipients based on an interferon-gamma-releasing assay: an exploratory randomized controlled trial. J Antimicrob Chemother. 2015;70(5):1567-72.

10. Kim SH, Lee SO, Park JB, Park IA, Park SJ, Yun SC, et al. A prospective longitudinal study evaluating the usefulness of a T-cell-based assay fo latent tuberculosis infection in kidney transplant recipients. Am J Transplant. 2011;11(9):1927-35.

11. Manuel O, Humar A, Preiksaitis J, Doucette K, Shokoples S, Peleg AY, et al. Comparison of quantiferon-TB gold with tuberculin skin test for detecting latent tuberculosis infection prior to liver transplantation. Am J Transplant. 2007:7(12):2797-801.

12. Chung WK, Zheng ZL, Sung JY, Kim S, Lee HH, Choi SJ, et al. Validity of interferon-gamma-release assays for the diagnosis of latent tuberculosis in haemodialysis patients. Clin Microbiol Infect. 2010;16(7):960-5.

13. Winthrop KL, Nyendak M, Calvet H, Oh P, Lo M, Swarbrick G, et al. Interferon-gamma release assays for diagnosing mycobacterium tuberculosis infection in renal dialysis patients. Clin J Am Soc Nephrol. 2008:3(5):1357-63.

14. Currie AC, Knight SR, Morris PJ. Tuberculosis in renal transplant recipients: the evidence for prophylaxis. Transplantation. 2010;90(7):695-704.

15. Slater ML, Welland G, Pai M, Parsonnet J, Banaei N. Challenges with QuantiFERON-TB Gold assay for large-scale, routine screening of U.S. healthcare workers. Am J Respir Crit Care Med. 2013;188(8):1005-10.

16. Vikrant S, Agarwal SK, Gupta S, Bhowmik D, Tiwari SC, Dash SC, et al. Prospective randomized control trial of isoniazid chemoprophylaxis during renal replacement therapy. Transpl Infect Dis. 2005;7(3-4):99-108.

17. Agarwal SK, Gupta S, Dash SC, Bhowmik D, Tiwari SC. Prospective randomised trial of isoniazid prophylaxis in renal transplant recipient. Int Urol Nephrol. 2004;36(3):425-31.

18. Sester M, Sotgiu G, Lange C, Giehl C, Girardi E, Migliori GB, et al. Interferongamma release assays for the diagnosis of active tuberculosis: a systematic review and meta-analysis. Eur Respir J. 2011;37(1):100-11.

19. Joo DJ, Kim BS, Kim SJ, Huh KH, Kim MS, Ju MK, et al. Risk factors and characteristics of post-transplant tuberculosis in an endemic area. Ann Transplant. 2013;18:163-73.

20. Canet E, Dantal J, Blancho G, Hourmant M, Coupel S. Tuberculosis following kidney transplantation: clinical features and outcome. A French multicentre experience in the last 20 years. Nephrol Dial Transplant. 2011:26(11):3773-8.

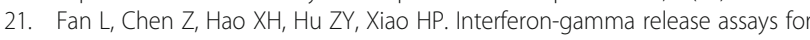
the diagnosis of extrapulmonary tuberculosis: a systematic review and meta-analysis. FEMS Immunol Med Microbiol. 2012;65(3):456-66.

22. Morris MI, Daly JS, Blumberg E, Kumar D, Sester M, Schluger N, et al. Diagnosis and management of tuberculosis in transplant donors: a donorderived infections consensus conference report. Am J Transplant. 2012;12(9):2288-300

23. Yang J, Lee J, Huh KH, Park JB, Cho JH, Lee S, et al. KNOW-KT (KoreaN cohort study for outcome in patients with kidney transplantation: a 9-year longitudinal cohort study): study rationale and methodology. BMC Nephrol. 2014:15:77.

\section{Submit your next manuscript to BioMed Central and we will help you at every step:}

- We accept pre-submission inquiries

- Our selector tool helps you to find the most relevant journal

- We provide round the clock customer support

- Convenient online submission

- Thorough peer review

- Inclusion in PubMed and all major indexing services

- Maximum visibility for your research

Submit your manuscript at www.biomedcentral.com/submit
Biomed Central 